\title{
The Cognitive Operator 4.0
}

\author{
Peter THORVALD ${ }^{\mathrm{a}, \mathrm{b}, 1}$, Åsa FAST BERGLUND ${ }^{\mathrm{b}}$ and David ROMERO ${ }^{\mathrm{c}}$

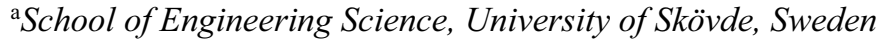 \\ ${ }^{\mathrm{b}}$ Department of Industrial and Materials Science, Chalmers University of Science, \\ Gothenburg, Sweden \\ ' Tecnológico de Monterrey, Mexico City, Mexico
}

\begin{abstract}
While previous Industrial Revolutions have increasingly seen the human as a cog in the system, each step reducing the cognitive content of work, Industry 4.0 contrarily views the human as a knowledge worker putting increased focus on cognitive skills and specialised craftsmanship. The opportunities that technological advancement provide are in abundance and to be able to fully take advantage of them, understanding how humans interact with increasingly complex technology is crucial. The Operator 4.0, a framework of eight plausible scenarios attempting to highlight what Industry 4.0 entails for the human worker, takes advantage of extended reality technology; having real-time access to large amounts of data and information; being physically enhanced using powered exoskeletons or through collaboration with automation; and finally real-time monitoring of operator status and health as well as the possibility to collaborate socially with other agents in the Industrial Internet of Things, Services, and People. Some of these will impose larger cognitive challenges than others and this paper presents and discusses parts of the Operator 4.0 projections that will have implications on cognitive work.
\end{abstract}

Keywords. Operator 4.0, Cognitive Work, Human Factors, Cognitive Operator 4.0.

\section{Introduction}

The Fourth Industrial Revolution is ever-present in most types of manufacturing sciences along with being under heavy focus from companies wanting to be competitive and take advantage of new and potentially revolutionising technological opportunities. Where previous Industrial Revolutions have inherently also transformed the nature of human work, the Fourth Industrial Revolution is no exception [1,2].

The First Industrial Revolution, taking off with the introduction of steam power and mechanisation as well as assisted and dextrous work, started a slow transition from a focus on crafts and craftsmanship organised in guilds, to the first modern factories (1761). Human knowledge and skills were still very much at the centre of manufacturing while the introduction of electrical power and mass-production during the Second Industrial Revolution towards the end of the $19^{\text {th }}$ century ultimately reduced the human to being a $\operatorname{cog}$ in the system and human work became more about performing single specialised and highly repetitive tasks in the production line [3]. The Third Industrial Revolution came in the mid- $20^{\text {th }}$ century and was the result of automation and computerization of work. The development of automation and robotics diversified roles among workers and created the need for monitoring while the desire for product customisation led to new demands on manual workers [1-3]. From a cognitive ergonomics perspective [4], new aspects of

\footnotetext{
${ }^{1}$ Corresponding Author. peter.thorvald@his.se
} 
study such as vigilance and attention theory were introduced from neighbouring fields. The Fourth Industrial Revolution, which we very much find ourselves in the midst of, has in many ways reverted to seeing the human as a knowledge worker again [2]. Onepiece flows lead to high demands on manual work and at the same time, the possibilities to support manual work through technology is increasing [1,2]. While Human Factors started as an attempt to fit "the human to the task" [5], we are finally at a stage where it is technologically feasible to turn the tables and "adapt technology to the human" [6-8].

\section{The Operator 4.0 - Typology}

Romero et al. [1,2] proposed the Operator 4.0, which is a framework of eight plausible scenarios attempting to highlight what Industry 4.0 entails for the human worker. These eight projections [2] include technology enhancing operators' physical and cognitive capabilities as it is likely that these augmentations will appear in the future, or indeed, as they already do [6-8]. The Operator 4.0 is also supported by the ACE Factories Cluster whitepaper focus on "Human-Centred Factories" [6] and is also one of the foundations for the European Commission brief on - Industry 5.0 - towards "Sustainable, HumanCentric and Resilient European Industry" [8].

The Super-Strength Operator visualizes the use of exoskeletons to enhance human strength. Exoskeletons, as they appear today, can be passive, using springs and wires, or they can be powered, using electrical motors, hydraulics or pneumatics to enhance biomechanical strength even further. The use of exoskeletons can allow for more flexibility and efficiency in manual work tasks, reduce work-related injuries, and can also help overcome one of the major challenges in the western industry today, namely that of a changing demographic and an ageing workforce [2]. The current status of this technology is not however without challenges as they often limit the range of motion, cannot fully cope with a huge diversity of anthropometrics, and can, if not used properly, result in pressure injuries [9].

The Augmented Operator takes advantage of the rapid development of Augmented Reality (AR) technology and wearables for allowing human workers real-time visual access to information in the form of visual overlays in smart glasses, tablets, smartphones, etc. There are several ways of presenting information through AR technology where smart glasses such as the Microsoft HoloLens or Google Glass might receive the most attention, but it is by no means the only way of augmenting reality [2]. Some of the first and still most effective ways of utilizing the technology are through smartphones or tablets [2] where several cases have applied this technology to maintenance and remote guidance for example [10].

The Virtual Operator, much like the augmented operator, deals with information visualisation and takes advantage of Virtual Reality (VR) technology for this. While AR focuses on overlaying information on the visual array, VR completely replaces the physical world with a virtual one. It simulates a fully immersive experience and allows a user to "step into" the virtual world where it can be used for factory planning, design considerations, or, as is most relevant from a cognitive perspective, virtual training [2].

The Healthy Operator makes use of the boom of wearable devices and bio-signal trackers that have become increasingly popular in later years. Several attempts at using both medicinal bio-signal equipment as well as off the shelf fitness trackers have shown promising results in allowing monitoring of a workers biological and cognitive status and wellbeing $[2,11]$. 
The Smarter Operator scenario is a result of the abundance of information available at the click of a button, or even of a text or voice command. Utilizing smartphones, personal assistants, and computers gives direct access to information previously distant. Having maintenance manuals, inventory lists, or task instructions available all the time and everywhere, potentially increases productivity and operational efficiency [2,12].

A Collaborative Operator is a human worker cooperating with collaborative robots (cobots) or other types of collaborative automation [2]. Cobots allow different levels of cooperation and collaboration between humans and cobots/automation ranging from coexistence to full collaboration in a shared physical space, which is challenging both due to safety aspects of humans interacting in close quarters with cobots as well as because of communication between these two agents: human and cobot [13].

The Social Operator is a human worker utilizing industrial social networks and enterprise social networking services to allow for collaboration, knowledge sharing, and knowledge transfer within social groups (incl. smart machines) in manufacturing [2,14].

The Analytical Operator, concludes the list of projections and, regards human workers having access to big data analytics to leverage data to drive decision-making for better forecasts, understanding of shop-floor performance, and greater visibility of both production status as well as other relevant KPIs [2].

Romero et al. [2] classify all these human-technology interactions but the superstrength operator and the collaborative operator to include cognitive interaction between the human workers and these human-centric Industry 4.0 technologies.

\section{The Cognitive Operator 4.0 - Potentials \& Challenges}

The degree of cognitive interaction involved in the eight human-technology interactions listed above varies to a considerable degree although the argument for its inclusion in all of them can easily be made. This section will outline in detail how cognitive interaction affects them, highlighting scientific and industrial potential as well as the challenges involved and questions that remain unanswered.

\subsection{Mental Workload}

There is a fundamental difference between classical ergonomics being mainly concerned with the body and how it is affected by work, and cognitive ergonomics also including the mental aspects and increasingly viewing the human as a cognitive agent that not only perceives the world but acts in it [4]. This relevant difference lies in the former being objectively observable and often measurable while the latter is not. If the problem of other minds [15] has taught us anything, it is that we cannot ever know what happens within the mind of someone else, ergo we cannot objectively measure their mental workload. What we can do, however, is assess subjective mental workload [16] or estimate mental workload or complexity of work from different rating scales $[17,18]$ Furthermore, there is potential for collecting bio-signal data corresponding with mental workload using wearables such as proposed by the Healthy Operator $4.0[2,11,19]$. The potential for such technology is great but also inflicted with large integrity issues as found by Kaasinen et al. [20], where manufacturing personnel voiced concern with this type of tracking technology. It is clear though, that with the increased focus on humans as knowledge workers rather than cogs in a production system, and human centricity being pointed out as one of the main pillars of Industry 5.0 [8], there is a need for better methods of assessing and understanding optimal levels of mental workload. 


\subsection{Communication}

With regards to the Collaborative Operator, Romero et al. [2] mainly saw cobots as a way of offloading physically demanding or unsafe tasks onto a robot sharing the same physical space as the worker. To be fair, most industrial collaborative applications have not come much further today and most certainly had not in the year 2016. However, the classifications of human-robot collaboration levels [13,21] do range from that type of independent co-existence in a shared space to full collaboration, meaning that the human and the robot can work on the same product at the same time with shared task allocation which does infer some kind of communication between the two. The robot (or the system rather) must be able to recognize what the human is doing, or even more importantly, is going to do and what space they will occupy in the near future to avoid confusion, conflicts, and even collision. And consequently, the robot must have a behaviour that can be predictive to the human user so as to avoid physical conflicts in the shared workspace [22]. A safety issue for sure but seeing as collaborative applications do have extensive safety procedures (e.g., ISO15066) implemented to guarantee safety, perhaps it becomes less about safety and more of a way to avoid unnecessary machine stops and optimize robot utilization.

Increasing access to (big) data and information as described in both the Analytical Operator $4.0 \&$ the Smarter Operator 4.0 [2] also creates a higher demand for properly designed information interfaces. User experience design in different forms has been an increasingly popular and potent topic of study since the introduction of the personal computer but applied research on data/information visualization in manufacturing is still fairly occasional and most often a result of initiatives by individual researchers or projects, never getting the kind of proliferation it needs and indeed deserves. For example, using AR technology to visualize task information requires new knowledge on how to design for AR and while there are novel attempts at investigating this, the field is very much in its infancy [23].

\subsection{Cognitive Embodiment}

Viewing exoskeletons and physical enhancement as mainly having to do with physical interaction might be reasonable given where the cognitive ergonomics field is largely influenced by theories from cognitive psychology where the separation of human factors into physical and mental aspects is still far too common. However, development in cognitive science during the past few decades have somewhat successfully started to question these assumptions and this argument is also finding its way into the applied ergonomics field [4, 24-26]. In essence, it is argued and demonstrated in many ways how our physical bodies not only affect how we cognize in the world but that it is necessary for cognition [26-30]. Changing the capabilities and limitations of the said physical body would therefore also change how that human interacts and cognizes with the world.

By its very nature, human cognition involves perception and action and is evolved to be most effective within a situation, here and now [26,31]. This does not mean that offline cognition does not happen, but that "even when decoupled from the environment, the activity of the mind is grounded in mechanisms that evolved for interaction with the environment - that is, mechanisms of sensory processing and motor control" [31]. So, where we cannot be situated, tools that simulate physical presence have proven very advantageous, such as the case of the Virtual Operator 4.0 [2]. Therefore, the use of VR tools to simulate ergonomics, to allow us to "step into" a factory before it is even built 
or to virtually simulate the placement of new equipment in shop floors, allows us to better utilize our cognition and ultimately make better and more informed decisions. For the same reasons, $V R$ also has great potential in the training of new personnel where it allows us to do realistic, offline training in a cost-efficient and safety-conscious way.

\subsection{A Working Definition for The Cognitive Operator 4.0}

Cognitive Interaction is critical to realize systems in which humans and technology work in symbiosis [32]. The Cognitive Operator 4.0 emerging term aims to stand for those knowledge workers and their cognitive interactions in which they exchange and jointly process information with the help of technologies to symbiotically perform a task (e.g., a physical action and/or a decision). This by combining computational and sensing technologies as well as interaction design to realize deep perception, awareness, and understanding between both collaborative agents.

\section{Discussion and Industry 5.0 Outlook}

Industry 5.0 - as described in a political brief from the European Commission (EC) will rest upon three main pillars: sustainability, resilience, and human-centricity [8], the last of which being heavily influenced by the Operator 4.0 vision [1,2]. The EC envisions large needs for education, training, re-skilling, and up-skilling as being some of the most pressing issues to address in the ongoing digital transformation of industry and society, making digital competencies a key factor. A precaution though, just because something involves humans, does not automatically mean that it is human-centred. Many initiatives sail under the flag of human-centricity while being more focused on the technology in itself. The key difference being that true human-centred approaches focus on the interaction and what can be achieved by human and technology in symbiosis [1].

The Operator 4.0 and human-centricity also responds to an even more pressing matter in western society, the challenge of changing demographics. An ageing workforce coupled with difficulties for the manufacturing industry to attract workers presents huge challenges with securing tomorrow's manufacturing [7]. Challenges that can, at least in part, be met by utilizing technology that allows for a more diverse industrial workforce and a technologically stimulating environment that raises the value of competence rather than physical fitness and youth [2,7].

So, ultimately the human worker of the future will have to be able to interact and communicate comfortably and efficiently with diverse technologies, they need to collect and consume large quantities of information, they need to transfer knowledge between virtual and real worlds and back again, and they need to be responsible not only for the continuing development of their own skills and knowledge but also for their colleagues. For this to happen and for us to be able to utilize the possibilities offered by technology, we need to know more about how to make these systems human-centred, and for that, the unit of study must be both the physical and the cognitive interaction between humans and technology, as well as the social and material context in which it emerges.

\section{References}

[1] D. Romero, P. Bernus, O. Noran, J. Stahre, and Å. Fast-Berglund, "The Operator 4.0: Human CyberPhysical Systems \& Adaptive Automation towards Human-Automation Symbiosis Work Systems," Production Management Initiatives for a Sustainable World, IFIP AICT 488, Springer, pp. 677-686, 2016. 
[2] D. Romero, J. Stahre, T. Wuest, O. Noran, P. Bernus, Å. Fast-Berglund, and D. Gorecky, "Towards an Operator 4.0 Typology: A Human-Centric Perspective on the Fourth Industrial Revolution Technologies," Int'l. Conf. on Computers and Industrial Engineering (CIE46), Tianjin, China, 2016, pp. 29-31.

[3] P. N. Stearns, The Industrial Revolution in World History, Routledge, 2020.

[4] E. Hollnagel, “Cognitive Ergonomics: It's All in the Mind," Ergonomics, 40(10):1170-1182, 1997.

[5] K.H. Kroemer, Fitting the Human: Intro. to Ergonomics/Human Factors Engineering. CRC Press, 2017.

[6] S. Zikos, G. Albanis, M. Tsourma et al., "Human-centred Factories from Theory to Industrial Practice. Lessons Learned and Recommendations," ACE Factories Cluster Whitepaper, October 2019.

[7] D. Romero, J. Stahre, and M. Taisch, "The Operator 4.0: Towards Socially Sustainable Factories of the Future,” Computers \& Industrial Engineering, Vol. 139, p. 106128, 2020.

[8] European Commission, "Industry 5.0: Towards a Sustainable, Human-Centric and Resilient European Industry," Whitepaper, 2021.

[9] E. Perez Luque, D. Högberg, A. Iriondo Pascual, D. Lämkull, and F. Garcia Rivera, "Motion Behavior and Range of Motionwhen Using Exoskeletons in Manual Assembly Tasks," 9th Swedish Production Symposium, Jönköping, Sweden, IOS Press, pp. 217-228, 2020.

[10] Å. Fast-Berglund, L. Gong, and D. Li, "Testing and Validating Extended Reality (xR) Technologies in Manufacturing," Procedia Manufacturing, 25:31-38, 2018.

[11] D. Romero, S. Mattsson, Å. Fast-Berglund, T. Wuest, J. Stahre, and D. Gorecky, D. "Digitalizing Occupational Health, Safety and Productivity for the Operator 4.0," Production Management for DataDriven, Intelligent, Collaborative, and Sustainable Mfg., IFIP AICT 536, Springer, pp. 473-481, 2018.

[12] R. Rabelo, D. Romero, and S.P. Zambiasi, "Softbots Supporting the Operator 4.0 at Smart Factory Environments," Production Management for Data-Driven, Intelligent, Collaborative, and Sustainable Manufacturing, IFIP AICT 536, Springer, pp. 456-464, 2018.

[13] A. Kolbeinsson, E. Lagerstedt, and J. Lindblom, "Classification of Collaboration Levels for HumanRobot Cooperation in Manufacturing," 16th Int'l. Conf. on Mfg. Research, Skövde, Sweden, IOS Press, $8: 151,2018$.

[14] D. Romero et al., "Social Factory Architecture: Social Networking Services and Production Scenarios through the Social Internet of Things, Services and People for the Social Operator 4.0," Shaping the Future of Production Management, IFIP AICT 513, Springer, pp. 265-273, 2017.

[15] D.C. Dennett, Brainstorms: Philosophical Essays on Mind and Psychology. MIT Press, 2017.

[16] S.G. Hart, "NASA-Task Load Index (NASA-TLX): 20 Years Later," Human Factors and Ergonomics Society Annual Meeting, Sage Publications, 50:904-908, 2006.

[17] S. Mattsson, M. Tarrar, and Å. Fast-Berglund, "Perceived Production Complexity-Understanding More than Parts of a System," Production Research, 54(20): 6008-6016, 2016.

[18] P. Thorvald, J. Lindblom, and R. Andreasson, "On the Development of a Method for Cognitive Load Assessment in Manufacturing," Robotics and Computer-Integrated Manufacturing, 59:252-266, 2019.

[19] S. Sun, X. Zheng, B. Gong, J. García Paredes, and J. Ordieres-Meré, "Healthy Operator 4.0: A Human Cyber-Physical System Architecture for Smart Workplaces,” Sensors, 20(7):2011, 2020.

[20] E. Kaasinen et al., "Empowering and Engaging Industrial Workers with Operator 4.0 Solutions," Computers \& Industrial Engineering, 139:105678, 2020.

[21] G. Michalos S. Makris, P. Tsarouchi, T. Guasch, D. Kontovrakis, and G. Chryssolouris, "Design Considerations for Safe Human-Robot Collaborative Workplaces," Procedia CIRP, 37:248-253, 2015.

[22] R.T. Chadalavada et al. "That's On My Mind! Robot to Human Intention Communication through Onboard Projection on Shared Floor Space," European Conference on Mobile Robots, IEEE, pp. 1-6, 2015.

[23] J. Blattgerste et al. "Comparing Conventional and Augmented Reality Instructions for Manual Assembly Tasks," 10th Int'l. Conf. on Pervasive Technologies Related to Assistive Environments, pp. 75-82, 2017.

[24] J.R. Wilson, "Fundamentals of Ergonomics in Theory and Practice," Applied Ergonomics, 31(6):557$567,2000$.

[25] J.R. Wilson, "Fundamentals of Systems Ergonomics/Human Factors," Applied Ergonomics, 45:5-13, 2014.

[26] J. Lindblom, and P. Thorvald, "Manufacturing in the Wild - Viewing Human-based Assembly through the Lens of Distributed Cognition," Production \& Manufacturing Research, 5(1):57-80.

[27] R. Andreasson, J. Lindblom, and P. Thorvald, "Distributed Cognition in Manufacturing - Collaborative Assembly Work," 14th Int'l. Conf. on Mfg. Research, Loughborough, UK, IOS Press, pp. 243-248, 2016.

[28] P. Thorvald, and J. Lindblom, "Distributed Cognition at Work - A Systemic Perspective on Humanbased Manufacturing," 13th Int'l. Conf. on Mfg. Research, Bath, UK, 2015.

[29] A. Kolbeinsson and J. Lindblom, "Mind the Body: How Embodied Cognition Matters in Manufacturing," Procedia Manufacturing, 3:5184-5191, 2015.

[30] J. Lindblom, Embodied Social Cognition, Berlin: Springer Verlag, 2015.

[31] M. Wilson, "Six Views of Embodied Cognition," Psychonomic Bulletin \& Review, 9(4):625-636, 2002.

[32] G. Jacucci, A. Spagnolli, J. Freeman, and L. Gamberini, "Symbiotic Interaction: A Critical Definition and Comparison to other Human-Computer Paradigms," Symbiotic Interaction, pp. 3-20, Springer, 2014. 